\title{
A terapia cognitivo-comportamental em grupo no Transtorno de Ansiedade Social
}

\section{Cognitive-behavioral group therapy in Social Anxiety Disorder}

\section{Terapia de grupo cognitivo-conductual en el trastorno de ansiedad social}

\section{Carolina Pereira Dittz*}

Universidade Federal de Juiz de Fora - UFJF, Juiz de Fora, Minas Gerais, Brasil

Francesca Stephan**

Universidade Federal de Juiz de Fora - UFJF, Juiz de Fora, Minas Gerais, Brasil

\section{Daniel Alexandre Gouvêa Gomes***}

Universidade Federal de Juiz de Fora - UFJ F, Juiz de Fora, Minas Gerais, Brasil

\section{Auxiliatrice Caneschi Badaró*}

Universidade Federal de Juiz de Fora - UFJF, Juiz de Fora, Minas Gerais, Brasil

\section{Lelio Moura Lourenço****}

Universidade Federal de Juiz de Fora - UFJ F, Juiz de Fora, Minas Gerais, Brasil

\begin{abstract}
RESUMO
O transtorno de ansiedade social (TAS), caracterizado pelo medo ou ansiedade acentuados em situações sociais, é capaz de comprometer significativamente a qualidade de vida do indivíduo. O estudo visou descrever o desenvolvimento de uma intervenção em grupo, baseada na Terapia Cognitivo-Comportamental, para pessoas que apresentaram os sintomas de TAS. O protocolo de tratamento utilizou as seguintes técnicas: reestruturação cognitiva, exposição às situações temidas, treino de habilidades sociais e relaxamento. O grupo foi formado por cinco voluntários acima dos 18 anos, da cidade de Juiz de Fora - Minas Gerais, os quais apresentaram pontuações elevadas em instrumentos de rastreio para o TAS. Foram realizados 16 encontros presenciais, com duração de 90 minutos. 0 estudo se dividiu em cinco fases: acolhimento, habituação, treinamento de habilidades, prevenção de recaída e finalização. Os resultados mostraram que os participantes tiveram melhoras, nos escores dos testes: Liebowitz, BAl e BDI (administrados antes e depois do tratamento) e nas observações clínicas realizadas, principalmente na forma de lidar com as situações sociais em pequenos grupos (participar e falar). Foi possível concluir que 0 protocolo de atendimento alcançou seus objetivos no tratamento do transtorno de ansiedade social. Entretanto, são necessárias alterações para que os resultados sejam provados mais satisfatoriamente.
\end{abstract}


Palavras-chave: terapia cognitivo-comportamental, terapia em grupo, transtorno de ansiedade social, fobia social.

\begin{abstract}
The social anxiety disorder (SAD), characterized by fear or excessive anxiety in social situations, is able to significantly impair the quality of life of an individual. The study aimed to develop a group intervention based on cognitive-behavioral therapy for people who showed symptoms of SAD. The treatment protocol has used the following techniques: cognitive restructuring, exposure to feared situations, social skills training, and relaxation. The group was composed by five volunteers, over 18, citizens of Juiz de Fora, Minas Gerais, which had high scores on screening instruments for TAS. Were conducted 16 sessions, during 90 minutes. The study was divided into five phases: reception, habituation, skills training, preventing relapse and completion. The results showed that the participants had improvements in test scores: Liebowitz, BAI and BDI (applied after and before the treatment) and clinical observations, especially in dealing with social situations in small groups (participating and speaking). It was concluded that the treatment protocol achieved its objectives in the treatment of social anxiety disorder, but changes are needed to the results if they prove more satisfying.
\end{abstract}

Keywords: cognitive-behavioral therapy, group therapy, social anxiety disorder, social phobia.

\title{
RESUMEN
}

El trastorno de ansiedad social (TAS) se caracteriza por el miedo o la ansiedad acentuada em situaciones sociales. Lo é capaz de deteriorar significativamente la calidad de vida del individuo. El objetivo del estudio fue desarrollar una intervención grupal basada en la terapia cognitivoconductual para las personas que mostraban síntomas de TAS. El protocolo de tratamiento se utiliza de las siguientes técnicas: reestructuración cognitiva, exposición a situaciones temidas, entrenamiento en habilidades sociales y de relajación. El grupo fue compuesto por cinco voluntarios, mayores de 18 años, de la ciudad de Juiz de Fora, Minas Gerais, que han tenido altas puntuaciones en los instrumentos de detección para TAS. Se llevaron a cabo 16 reuniones, con duración de 90 minutos. El estudio se divide en cinco fases: recepción, habituación, entrenamiento, la prevención y la terminación. Los resultados mostraron que los participantes han tenido una mejora en las puntuaciones de las pruebas: Liebowitz, BAl y BDI (aplicado antes y después del tratamiento) y observaciones clínicas, principalmente en el tratamiento de las situaciones sociales en grupos pequeños (participar y hablar). Se ha concluido que el protocolo de tratamiento logró sus objetivos en el tratamiento del trastorno de ansiedad social, pero se necesitan cambios en los resultados si prueban más satisfactoria.

Palabras clave: terapia cognitivo-conductual, terapia de grupo, trastorno de ansiedad social, fobia social.

\section{I ntrodução}

O Transtorno de Ansiedade Social (TAS) é definido como medo ou ansiedade clinicamente significativa, provocados pela exposição a certos tipos de situações sociais, que podem ser de desempenho, de 
observação ou de interação, segundo o Manual Estatístico e Diagnóstico de Transtornos Mentais (DSM), 5o edição, publicado pela Associação Americana de Psiquiatria (APA, 2014). Até o DSM-IV-TR (Associação Americana de Psiquiatria, 2002) este transtorno era reconhecido predominantemente pela nomenclatura de fobia social, termo que pode ser encontrado até os dias atuais, em trabalhos realizados e publicados em vários locais do mundo. No presente trabalho, os dois termos, TAS e fobia social, são considerados sinônimos na descrição do quadro ansioso específico.

O TAS foi por certo tempo um transtorno negligenciado (Liebowitz, Gorman, Fyer, \& Klein, 1985; Nutt \& Ballenger, 2010), no entanto, atualmente, passou a ser reconhecido como um problema psiquiátrico crônico associado a um considerável comprometimento funcional, podendo ser incapacitante em suas formas mais graves (Barlow, 1999; Barlow, 2002; Nutt \& Ballenger, 2010). Para aquelas pessoas que apresentam o transtorno e que chamaremos aqui de ansiosos sociais, as situações sociais, de um modo geral, são evitadas ou toleradas com intenso sofrimento e ansiedade, com sintomas como palpitações, tremores, sudorese, desconforto gastrointestinal, diarréia, tensão muscular e rubor facial.

Segundo Knijnik (2008), a etiologia da ansiedade social se dá pela interação de fatores genéticos, biológicos, cognitivos, comportamentais e psicodinâmicos. A compreensão das origens deste transtorno, auxilia na percepção dos fatores que corroboram para o desenvolvimento da ansiedade social e possibilita o aprimoramento de novas medidas de prevenção (Hudson \& Rapee, 2000). Portanto, mais pesquisas sobre o tema são necessárias para a compreensão das variáveis envolvidas.

Estima-se que o distúrbio de ansiedade social seja alto na população, com prevalência entre $5 \%$ e $13 \%$ da população geral, que resultam em diferentes graus de incapacidade e limitações sociais e ocupacionais (Nardi, 2000; Picon, 2006). Segundo o National Comorbity Survey (NCS) a prevalência da fobia social ao longo da vida é de 12,1\% (Kessler et al., 2005). Nos países ocidentais tem-se estimado a prevalência do transtorno de ansiedade social entre 7 e 13\% (Furmak, 2002). Segundo Pereira e Lourenço (2012), o Brasil ainda não realizou estudos epidemiológicos representativos da população a respeito da prevalência da ansiedade social, o que demonstra a necessidade em compreender mais sobre o transtorno em nossa realidade. Apesar deste transtorno interferir na qualidade de vida, tantos os profissionais de saúde, quanto aos ansiosos sociais têm dificuldades de identificar o TAS. Muitas das vezes o tratamento é procurado depois de 15 anos de convivência com os sintomas (Wang et al, 2005).

Conforme apontado pelo NCS $81 \%$ dos pacientes com ansiedade social apresentam algum tipo de comorbidade (Kessler et al., 2008), 
sendo detectadas como mais frequentes a sua associação com a fobia simples, abuso ou dependência de álcool e depressão maior (Knijnik, Kruter, Cordioli, \& Kapczinski, 2005). A porcentagem de casos comórbidos com a ansiedade social é mais elevada entre os indivíduos que se enquadram em um maior número de situações sociais temidas (Ruscio et al., 2008).

Atualmente, as abordagens terapêuticas empiricamente testadas e reconhecidas como eficazes no tratamento da ansiedade social são a farmacoterapia e a terapia cognitivo-comportamental individual (TCCI) e em grupo (TCCG) (Ito, Roso, Tiwari, Kendall, \& Asbahr, 2008). A Terapia Cognitivo-Comportamental (TCC), criada por Aaron Beck em 1956, supõe que o pensamento das pessoas afeta o que elas sentem e fazem. A maneira como cada um pensa está pautada em crenças que vão sendo criadas e reforçadas ao longo da vida, seja por experiências pessoais ou aprendida nas relações interpessoais (Wright, Basco, \& Thase, 2008). Beck (1997) organiza essas crenças em visões e pressupostos criados a respeito de si mesmo, dos outros, do mundo e do futuro e a partir disso são definidas regras e suposições que moldam a postura da pessoa em cada situação.

Por sua vez, a TCCl é educativa e de natureza focal. Prioriza as discussões práticas realizadas nas sessões e nas tarefas de casa (I to et al., 2008). O terapeuta tem postura colaborativa e ativa no tratamento, buscando auxiliar os pacientes na identificação de pensamentos prejudiciais e a responder a eles, de maneira funcional (Neufeld, Moreira, \& Xavier, 2012).

O trabalho em grupo tem sido referenciado como apropriado e vantajoso no tratamento do Transtorno de Ansiedade Social, por proporcionar ao indivíduo o relacionamento com outras pessoas do grupo, ou seja, o contato social por si só é estimulado diminuindo o isolamento (Hofmann, 2004). D'el Rey, Greenberg, Husni e Cejkinski (2007) desenvolveram uma pesquisa a fim de verificar a efetividade da terapia cognitivo-comportamental em grupo, em 29 sujeitos com transtorno de ansiedade social generalizada. A amostra, dividida em dois grupos (intervenção e controle), apontou para melhoras superiores no grupo que recebeu a intervenção baseada nas técnicas cognitivas e/ou comportamentais.

A psicoterapia em grupo com ansiosos sociais parece apresentar mais benefícios, como relatado por Picon e Knijnik (2008). Segundo esses autores, normalmente, os ansiosos sociais não compartilham seus medos e acreditam serem os únicos com os problemas que apresentam. As exposições, difíceis de serem recriadas na clínica individual, são geradas pelo grupo, o que favorece a reestruturação cognitiva. Para o transtorno de ansiedade social o grupo possui três componentes elementares: exposição na sessão, reestruturação cognitiva e tarefas de casa, sendo a primeira parte essencial do tratamento, responsável por romper com o ciclo de ansiedade social. 
Além disso, é relevante que as intervenções cognitivas ocorram sempre antes, durante e após cada exposição. Assim, um dos objetivos da terapia em grupo é fazer o paciente tornar-se o seu próprio terapeuta, para que o mesmo consiga enfrentar as situações ansiogênicas (Picon \& Knijnik, 2008).

Estudos indicam que, para o transtorno de ansiedade social, 12 a 20 sessões semanais em grupo são suficientes para a redução significativa da sintomatologia. A resposta ao tratamento também depende do número de comorbidades e da gravidade dos sintomas, sendo que no caso de existirem comorbidades o tratamento, em geral se torna mais prolongado. A terapia em grupo apresenta algumas vantagens em relação à terapia individual, visto que o grupo funciona como uma situação social vivida pelo paciente e observada pelo terapeuta. Sendo assim, o grupo é uma forma de exposição contínua e pode tornar mais fácil e realista a execução de situações práticas propostas (Nutt \& Ballenger, 2010).

A terapia cognitivo-comportamental em grupo (TCCG) foi amplamente utilizada no presente estudo, que utilizou o formato da TCCG descrito por D'el Rey et al. (2007) e seu grupo de pesquisa, baseado no protocolo de tratamento utilizado na Temple University por Heimberg (2002). Nesse modelo foram integradas técnicas de exposição e reestruturação cognitiva, apropriadas a esse modelo de tratamento. Em relação ao treinamento de habilidades sociais (THS), o mesmo foi utilizado, direta e indiretamente na exposição in(ao) vivo (às situações temidas).

Hope, Heimberg e Turk (2012), desenvolveram outro modelo cognitivo, que foca em como ansiedade social se mantém. Eles defendem que, ao estar em uma situação social, os indivíduos que apresentam sintomas de TAS se sentem como alvo de críticas em potencial e começam a criar imagens mentais sobre as representações que os outros estão criando deles. Isso é montado a partir da memória de longa duração, sinais internos (rosto afogueado, tremor nas mãos) e sinais externos (expressão facial dos outros, risos que podem surgir de alguém); junto a isso podem surgir imagens espontâneas de lembranças da época em que o transtorno estava se desenvolvendo. Dessa forma, observa-se que o indivíduo com sintomas de TAS é mais preocupado com a imagem transmitida do que os que não apresentam tais sintomas, e por isso a memória acessada durante uma exposição será sempre de um contexto ameaçador.

Diante disso, a pessoa começa um processo de automonitoração e busca exagerada por feedbacks dos demais (como movimento de sobrancelhas, movimentos com a boca, entre outro). Muitas vezes sua percepção e imagem criada do self encontram-se distorcidas em relação ao contexto real, confirmando cada vez mais suas crenças negativas. Todo esse processo cognitivo leva o indivíduo a utilizar 
mecanismos pouco adequados de enfrentamento ou até mesmo a evitar cada vez mais as situações sociais.

Esse modelo da ansiedade social propõe alguns pontos importantes para o tratamento desses transtornos, tais como: crenças negativas sobre os outros e sobre as situações sociais, crenças negativas sobre si mesmo, previsões negativas sobre os resultados de uma situação social, evitação associada a essas previsões negativas, foco de atenção em pistas de ameaça social em situações sociais, avaliação negativa do desempenho depois da situação social enfrentada. $O$ tratamento busca romper esse ciclo vicioso da ansiedade, ao trabalhar esses pontos expostos (Hope et al, 2012).

O presente estudo tem por objetivo, descrever os encontros dos grupos terapêuticos, baseados no protocolo de Heimberg (2002), mas organizados de acordo com as necessidades e o perfil dos participantes. Através dessa descrição, foi possível realizar uma análise das diferenças qualitativas, relativas aos sintomas dos participantes, e quantitativas, baseada nos escores dos pré e pós testes.

\section{Método}

A pesquisa, que corresponde a um estudo quase experimental, teve como ponto de partida o projeto intitulado "A efetividade da Terapia cognitivo-comportamental de grupo em pacientes com fobia social: pesquisa e extensão" que se enquadra nas atividades desenvolvidas por Núcleo de Estudos em Violência e Ansiedade Social (NEVAS), juntamente com a colaboração financeira da Fundação de Amparo à Pesquisa do estado de Minas Gerais (FAPEMIG). Tal núcleo consiste em um grupo de estudo, pesquisa e extensão, que se destaca pelos trabalhos direcionados aos campos de intervenção comunitária e clínica nas temáticas de violência, violência doméstica, bullying, contextos sócio-educativos, avaliação psicológica e ansiedade social.

O projeto em questão busca oferecer atendimento clínico baseado no TCCG à comunidade acima de 18 anos, advinda do Departamento de Saúde Mental da Secretaria Municipal de Saúde do município, do Centro de Pesquisa Aplicada (CPA) e de clínicas particulares. A equipe coordenadora do grupo foi composta por uma mestranda, que assumiu a função de terapeuta, e duas psicólogas, sendo uma delas responsável pelo papel de coterapeuta e a segunda pelas anotações necessárias para posterior análise do andamento das sessões e resultados das intervenções.

O projeto foi amplamente divulgado na comunidade com o nome de "Grupo de Tímidos" e os interessados realizaram suas inscrições no CPA, onde foram solicitadas informações necessárias à equipe, tais como nome, idade, telefone e disponibilidade de horário para as 
reuniões semanais. Em seguida a equipe entrou em contato com os interessados para agendar uma entrevista inicial, a qual consistiu na aplicação de instrumentos de rastreio como parte da avaliação inicial. Para a triagem dos participantes do grupo foi utilizada uma entrevista clínica padrão da instituição e as escalas Liebowitz, BAl e BDI. Essas mesmas três escalas foram utilizadas como formas de avaliação pré e pós-tratamento. Foram excluídos os participantes com apresentavam no BDI dados compatíveis com alguma comorbidade (transtornos psicóticos, abuso de substâncias), a qual poderia enviesar os resultados.

\subsection{Instrumentos}

Foram utilizados para obtenção dos resultados da pesquisa os dados sobre o desenvolvimento das sessões, obtidos através de observação e anotações de campo de informações específicas das sessões, como técnicas utilizadas, as quais forneceram as informações analisadas qualitativamente.

Os dados de natureza quantitativa foram obtidos através das seguintes escalas de autopreenchimento:

a) Escala de Ansiedade Social de Liebowitz - Visa avaliar situações referentes a interações sociais e de desempenho que os indivíduos com fobia social temem ou evitam. A escala é composta por 24 itens, do tipo Likert com 4 opções de respostas, que avaliam a gravidade atual da ansiedade e evitação em cada um dos itens (Masia-Warner et al.,2003). O resultado é alcançado através da soma das respostas e é classificado como: Fobia Social Moderada (55-65); Fobia Social Média (66-88); Fobia Social Grave (81-95) e Fobia Social Muito Grave (> 95). A escala foi utilizada em sua versão auto-aplicada (Santos, 2012).

b) Inventário de ansiedade de Beck (BAI) - Escala do tipo Likert destinada a medir o nível de ansiedade através de 21 itens, que avaliam a presença e a intensidade dos sintomas ansiosos. A pontuação do teste pode indicar o grau mínimo de ansiedade (de zero a dez pontos); ansiedade leve, (de 11 e 19 pontos); ansiedade moderada, (entre 20 e 30); e ansiedade grave (entre 31 e 63 pontos) (Cunha, 2001).

c) Inventário de depressão de Beck (BDI) - Escala do tipo Likert, com vinte e um grupos de afirmações, é destinada a medir a intensidade da depressão. Os escores de pontuação podem variar da seguinte forma: 0 a11, nível mínimo de depressão; 12 a 19, depressão leve; 20-35, depressão moderada; e 36-63 depressão severa (Cunha, 2001).

Através destas escalas e de uma ficha de Triagem Padrão disponibilizadas pelo CPA, que permite uma caracterização sociodemográfica dos participantes, identificamos o nível de 
ansiedade social, bem como as possíveis comorbidades que podem inviabilizar o tratamento.

\subsection{Procedimentos}

Após a avaliação dos instrumentos e a seleção dos participantes iniciaram-se os 16 encontros semanais, de noventa minutos, embasados no método de tratamento da terapia cognitivocomportamental. O grupo foi composto inicialmente por nove integrantes (quatro homens e cinco mulheres), porém um total de cinco participantes (três homens e duas mulheres) concluiu o tratamento proposto pela equipe. Dois participantes (mulheres) desistentes afirmaram não se sentiram confortáveis ao relatar suas experiências do dia a dia para o restante do grupo.Os outros dois participantes (um homem e uma mulher) desistiram do processo sem uma justificativa. Ao término da intervenção os participantes responderam a avaliação final com intuito de verificar se a intervenção foi eficaz na diminuição dos níveis dos sintomas de ansiedade. Essa fase contou com a aplicação dos mesmos questionários utilizados na avaliação inicial.

\subsection{Aspectos éticos}

A pesquisa foi submetida ao Comitê de Ética e Pesquisa e recebeu parecer favorável para sua execução, conforme apontado pelo Certificado de Apresentação para Apreciação Ética (no 26017214.9.0000.5147). Ressalta-se que para aqueles indivíduos que não se sentiram a vontade na intervenção em grupo, ou que apresentaram morbidades associadas ao quadro de transtornos de ansiedade social, foram oferecidos tratamento individual nos mesmos parâmetros terapêuticos do grupo.

\section{Resultados}

Como resultados deste estudo serão descritas inicialmente as fases das sessões realizadas, que representam a parte qualitativa dos dados e em seguida apresentados os resultados dos instrumentos antes e após as intervenções terapêuticas.

As sessões propostas pela equipe utilizaram um conjunto de técnicas cognitivas e comportamentais para o tratamento da ansiedade social, as quais buscam auxiliar na diminuição da sintomatologia nas situações sociais temidas. Esta intervenção foi baseada nas premissas discutidas por Heimberg (2002), e foram consideradas como pontos chave para o tratamento do transtorno de ansiedade social. São elas: 
a exposição, a reestruturação cognitiva, as técnicas de relaxamento e o treino de habilidades sociais.

Para realização das sessões, foram utilizadas técnicas cognitivas e comportamentais que atendem aos pressupostos básicos da TCCG para tratamento da ansiedade social. As sessões foram agrupadas em quatro fases, que contém a descrição do que foi trabalhado, além de momento de finalização, feedback e prevenção de recaídas.

\subsection{Fase de acolhimento}

Nesta fase os pacientes que aceitaram participar da pesquisaintervenção, tiveram o primeiro contato com os outros membros do grupo terapêutico. Este é um momento muito importante, por se tratar de uma exposição em grupo, que é uma das principais dificuldades relatadas pelos ansiosos sociais. A técnica inicial para facilitar a apresentação dos participantes e dos terapeutas foi a dinâmica nomeada como "quebra-gelo", que propicia um ambiente mais favorável ao início do grupo com o conhecimento dos nomes entre a equipe e os integrantes. Para a realização desta técnica dividiu-se o grupo e as psicólogas em duplas, assim cada um dos integrantes teve que apresentar seu par. Foi-lhes dado um momento para que pudessem conversar e falar mais sobre suas características, qualidades, dentre outros assuntos.

Ao final da apresentação, foi sugerido aos participantes que estabelecessem metas e objetivos que desejavam alcançar com o tratamento, para que os terapeutas pudessem direcionar as técnicas e orientar as sessões, maximizando assim a motivação e a participação dos integrantes nos encontros. Este processo é característico da TCCG (Heimberg, 2002) e deve ser realizado com a colaboração de todos os membros do grupo.

A psicoeducação também esteve presente no protocolo de tratamento e consistiu em ofertar experiências de ensino, para aumentar a eficácia do atendimento e reforçar uma prevenção de recaídas após o término da intervenção. Segundo Wright et al. (2008), o aprendizado de habilidades para mudança das cognições, estados de humor e de comportamento devem instrumentalizar o paciente com conhecimentos que auxiliam na redução dos riscos de reincidência dos sintomas. Neste aspecto, foram oferecidos ao grupo materiais, formulados pela equipe, apresentados em forma de textos com a finalidade de contextualizar o paciente sobre o modelo de tratamento baseado na TCCG, as principais distorções cognitivas, os sintomas da ansiedade e as características do transtorno de ansiedade social.

\subsection{Fase de habituação}


Neste momento foram introduzidos conceitos relacionados à reestruturação cognitiva e à exposição a situações sociais. As técnicas utilizadas exigiam a participação dos indivíduos de maneira ativa no grupo.

Primeiro foi realizada a técnica da balança, que tem por objetivo verificar os prós e os contras quando se tem que iniciar e/ou manter uma conversa. Assim, os participantes foram incentivados a preencher uma tabela que continha de um lado as vantagens, e, do outro, as desvantagens da situação proposta. Neste caso os terapeutas mediavam a tarefa e contribuíam para a reestruturação cognitiva, visto que algum ponto negativo poderia se transformar em positivo através da orientação profissional.

O registro de pensamento disfuncional foi apresentado ao grupo e proporcionou aos integrantes o direcionamento de sua atenção sobre as cognições importantes e os pensamentos automáticos. Ele também estimulou a indagação ao que se refere à validade dos modelos de pensamento dos pacientes (Wright et al.,2008). O registro foi entregue aos participantes, conforme o modelo apresentado por Beck (1997), e Ihes foi ensinado como preenchê-lo.

Para trabalhar com a exposição a situações sociais, foi utilizada a técnica do barbante, que consistia em cortar pedaços de barbantes do mesmo tamanho e pedir que cada integrante da dupla segurasse uma das pontas. A atividade era montar duplas que trançassem 0 barbante, formando um emaranhado. Através da cooperação do grupo o nó deveria ser desfeito e a equipe conseguia observar como os ansiosos sociais reagiam no grupo, além de habilidades de liderança, comunicação através das sugestões dos colegas para a resolução das questões, a assertividade ao não aceitar a sugestão, e outras tantas questões que surgem neste contexto.

Para reforçar a necessidade de desenvolver contato com outras pessoas foi sugerido ao grupo que pesquisasse, como tarefa de casa, algumas músicas e poesias para serem apresentadas na próxima sessão. No encontro seguinte cada integrante leu o texto selecionado e explicou o porquê da realização daquela escolha. Para os indivíduos que não levaram a tarefa foram oferecidos materiais similares àqueles pedidos, para que pudessem participar e contribuir para que a atividade fosse realizada com sucesso. Os sintomas ansiosos mais transparentes, como sudorese, rubor, queimação e taquicardia foram listados pelos sujeitos, no momento de realização da atividade. Isso serviu para auxiliar a psicoeducação a respeito dos sintomas ansiosos e pensamentos disfuncionais.

Nesta categoria apresentou-se o relaxamento muscular progressivo, o qual corresponde a um exercício de tensão e relaxamento dos principais grupos musculares (Manfro, Heldt, Cordioli, \& Otto, 2008). A técnica foi descrita para os participantes, que se deitaram em um colchonete durante a realização da técnica. Este exercício consiste em 
oferecer suporte ao sujeito, para que este perceba os sintomas fisiológicos que ocorrem, durante as situações sociais, e para que possa através da técnica, diminuir o seu grau de ansiedade.

Com a finalidade de desenvolver uma hierarquia de situações temidas, foi realizada a organização de uma lista de exposição, de forma gradual a fim de superar a ansiedade progressivamente (Wright et al., 2008). Foi pedido para que os participantes desenvolvessem uma lista de situações temidas e graduassem a ansiedade desencadeada por cada um dos itens. A classificação foi organizada de forma que os itens com menor grau de desconforto assumissem as primeiras colocações da hierarquia e assim progressivamente. Os participantes foram incentivados a executar as tarefas apresentadas na listagem ao longo das sessões.

A revisão do registro de pensamento disfuncional foi uma tarefa constantemente revisada em todas as sessões, visto que através dela identificamos pensamentos disfuncionais e facilitamos o processo de melhora entre os participantes, que passam a se expor as situações temidas com maior facilidade. A psicoeducação sobre a assertividade se fez presente nesta etapa. Um material foi entregue a cada membro apresentando a diferenciação entre a comunicação assertiva, agressiva e passiva. A comunicação assertiva recebeu destaque nesta etapa que foi descrita como "a expressão direta dos próprios sentimentos, necessidades, direitos legítimos ou opinião sem ameaçar ou castigar os demais e sem violar os direitos dessas pessoas" (Caballo, 2012, pág.361). Neste caso, a atividade em dupla foi destinada a diferenciar os tipos de comunicação, por isso foram oferecidas algumas situações do dia a dia para que eles respondessem a mesmas utilizando os diferentes tipos de comunicação.

\subsection{Fase de treinamento de habilidades}

A fim de estimular o treino da assertividade (Pureza, Rusch, Wagner, $\&$ Oliveira, 2012) aplicou-se uma técnica intitulada pela equipe como "Treino do elogio". Dividiu-se o grupo em duplas e cada parceiro sorteou uma qualidade, predefinida pelas terapeutas, e fez o elogio para seu parceiro usando a palavra sorteada. Para a outra pessoa, a técnica consistia em reconhecer qual era a palavra e falar sobre o elogio recebido. Todos os integrantes participaram e conseguiram concluir a tarefa com êxito.

Outra dinâmica realizada foi a "dinâmica do alfabeto", que teve como objetivo estimular a comunicação entre os participantes. As letras do alfabeto foram sorteadas e cada dupla deveria iniciar e manter a conversa utilizando uma letra do alfabeto para compor a primeira palavra emitida na conversa. Durante o feedback, os participantes dos grupos concluíram que no cotidiano existe menos limitação para 
se manter uma conversa, assim, para eles o medo neste contexto passou a ser exagerado.

\subsection{Finalização e prevenção de recaídas}

Para finalizar a intervenção, aplicou-se a técnica nomeada de "Bombardeio de elogios". Nesta, cada participante teve que ficar no centro de uma roda formada pelo grupo e os outros integrantes começaram a "bombardeá-lo" com elogios emitidos de forma desordenada e em grande número. Esta técnica foi realizada para deixar o participante em uma posição que se sentisse desconfortável, porém corriqueira no dia-a-dia. Neste momento foi pedido que os participantes realizassem um feedback sobre a intervenção e foi distribuído um texto com foco na prevenção de recaídas.

Ao final do processo foi possível realizar uma comparação entre os escores obtidos pelo uso das escalas nas etapas de avaliação anterior às sessões do grupo terapêutico e após a realização das mesmas. Seguem na tabela 1 abaixo os escores brutos de todos os participantes que concluíram o grupo terapêutico.

Tabela 1

Escores brutos da avaliação inicial e final

\begin{tabular}{ccccc}
\hline & \multicolumn{2}{c}{ Avaliação inicial } & \multicolumn{2}{c}{ Avaliação final } \\
\cline { 2 - 5 } Participantes & BAI & Liebowitz & BAI & Liebowitz \\
\hline 1 & 13 & 57 & 11 & 42 \\
2 & 3 & 68 & 5 & 62 \\
3 & 36 & 106 & 18 & 106 \\
4 & 16 & 61 & 12 & 53 \\
5 & 12 & 117 & 16 & 85 \\
\hline
\end{tabular}

Ao analisar a tabela acima, percebe-se que, no que diz respeito ao Inventário de Ansiedade de Beck, os pacientes 1, 3 e 4 obtiveram escores brutos menores na avaliação final do que na inicial, e os pacientes 2 e 5 tiveram um aumento em seus resultados em comparação à aplicação anterior. Apesar da diminuição nos escores, apenas o paciente 3 e 4 tiveram redução no grau de ansiedade. 0 paciente 3 passou de um nível de "Ansiedade Severa" para "Ansiedade moderada". A redução no escore bruto da escala foi de 36 para 18, ou seja, o paciente obteve como resultado, metade dos pontos, comparado à primeira avaliação. Na avaliação inicial foi 
marcada que ele se sentia incomodado "gravemente" pelos sintomas apresentados nos itens $4,5,7,20$ e 21 . Já na avaliação final não houve nenhuma sintomatologia, onde foi relatado incômodo grave, por parte das respostas do paciente, sendo que alguns dos itens foram considerados absolutamente incapazes de incomodar, como, por exemplo, o rosto afogueado e o suor (não devido ao calor). 0 participante 4, passou de um grau de "Ansiedade Moderada" para "Ansiedade Leve". Vale salientar que na primeira avaliação, ele estava no limiar entre os dois níveis, podendo o escore ser considerado "supervalorizado", por influência de alguma variável externa, visto que o instrumento rastreia apenas os sintomas de ansiedade apresentados nos últimos sete dias.

O paciente 2 manteve-se em um grau de "Ansiedade Mínima", enquanto o 5 subiu de "Ansiedade Leve" para "Ansiedade Moderada". Esta mudança se deu pela elevação de quatro pontos no preenchimento da escala. Da mesma forma que o paciente 4, estes resultados poderiam ser considerados influenciados por variáveis externas.

Sobre a Escala de Ansiedade de Liebowitz, percebe-se que quatro dos cinco participantes obtiveram escores menores na avaliação final, após a intervenção, e em um caso não houve alteração no resultado final. Os pacientes 1, 2, 4 e 5 obtiveram redução nos escores brutos. Uma análise das respostas fornecidas pelos participantes, sobre 0 medo ou ansiedade e a evitação das situações sociais expressas nos itens da escala foi realizada. Percebeu-se que alguns itens sofreram redução na forma como os indivíduos se sentiam. Eles não apresentavam mais medo ou ansiedade intensa diante das situações e não as evitavam com a mesma frequência o que faziam anteriormente.

No que diz respeito à classificação dos participantes quanto ao nível de ansiedade social experimentado na avaliação inicial e final, os resultados foram os seguintes: o paciente 1 saiu de "Fobia Social Moderada" para "Fobia Social Leve"; o 2 foi de "Fobia Social Média" para "Fobia Social Moderada"; o 3 permaneceu na mesma faixa de classificação sendo categorizado com sintomatologia de "Fobia Social muito grave"; o 4 reduziu seu nível de ansiedade de "Fobia Social Moderada" para "Fobia Social Leve" e o 5 teve uma diminuição na classificação de "Fobia Social muito grave" para "Fobia Social Grave". Este último paciente teve a diminuição na intensidade de experimentação dos sintomas em, pelo menos, nove itens da escala, tanto no que diz respeito ao medo ou ansiedade quanto à evitação, das situações sociais ou de desempenho. Essas avaliações se basearam na análise quantitativa dos escores, dos instrumentos aplicados antes e após o grupo terapêutico. A diminuição desses resultados está relacionada aos sintomas, que é o modelo em que os itens da escala foram construídos. 
A partir desses resultados, procedeu-se a uma análise qualitativa, com a utilização de uma metodologia de observação clínica dos participantes. Através destas, observou-se uma melhora nas atividades que envolviam a técnica de exposição, pois os pacientes relatavam uma queda no nível de ansiedade e consequentemente uma facilidade maior em desempenhar tarefas que demandavam o contato social. Tal conclusão foi alcançada como depoimento dos pacientes, que semanalmente apresentavam na sessão suas principais dificuldades e avanços no processo, através da discussão do registro de pensamento disfuncional. Ao longo do tratamento as atividades propostas ao grupo demandaram maior esforço, devido ao grau de ansiedade mais elevado, entretanto a equipe se surpreendeu como desempenho dos pacientes. Algumas dificuldades agrupadas de maneira hierárquica foram propostas, no entanto alguns participantes realizaram as tarefas de forma antecipada, ou reagiram de forma adaptativa, sem esquiva e com redução de pensamentos automáticos disfuncionais, em situações do dia a dia. Através da observação clínica observaram-se também avanços como a voz menos trêmula e com melhor entonação, facilidade em iniciar/manter a conversa e menor rubor facial ao relatar questões para o grupo.

\section{Discussão}

O programa de intervenção, realizado no presente trabalho, esteve de acordo com a proposta de Heimberg (2002), no que diz respeito aos focos principais da TCC, para o trabalho em grupo com pacientes com TAS. Foram realizadas atividades que trabalharam com exposição a situações sociais. Tais tarefas foram experimentadas pelos pacientes com alto grau de medo ou ansiedade e evitação, necessitando assim de uma busca constante de reestruturação cognitiva visando à diminuição dos pensamentos disfuncionais. Além disso, forma incluídos exercícios de relaxamento, para ensiná-los a lidar com os sintomas fisiológicos da ansiedade, e treino de habilidades sociais.

Os valores alcançados na Escala de Ansiedade Social de Liebowitz, com a diminuição dos sintomas de $80 \%$ da amostra, apontam que a TCCG apresentou indícios de melhora na qualidade de vida dos indivíduos. Por exemplo, quando questionados sobre como foi participar de um pequeno grupo de pessoas, os resultados do pósteste mostraram que o nível de medo ou ansiedade, experimentado por eles, passou de "Intenso" para "Leve" ou "Nenhum", comprovando que a exposição às situações sociais provoca uma reestruturação cognitiva a respeito do temor dos sujeitos. Outro ponto importante nesta situação, foi que a evitação da situação acima também diminuiu, passando para algo ocasional, ao invés de em 
todos os momentos. Mais uma situação que obteve escores satisfatórios foi o "Agir, realizar ou falar em frente a uma audiência", no qual o escore sofreu uma redução no grau de medo ou ansiedade e evitação, alcançando os mesmos efeitos da situação anterior. Nas demais situações os efeitos foram mais discretos, porém quando se olha o nível de medo ou ansiedade e evitação, alcançado pelos pacientes, percebe-se que há uma diminuição, favorecendo o desenvolvimento de novas habilidades de enfrentamento das situações.

As situações que foram o foco das intervenções e avaliação inicial e final foram baseadas no instrumento Liebowitz, haja vista sua oferta de situações sociais bem definidas e abrangentes. Contudo, durante o tratamento foram trabalhadas também demandas expostas pelo próprio paciente, mais específico com o cotidiano de cada paciente ( presente de forma mais geral na escala citada).

Um ponto positivo que foi comumente destacado pelos terapeutas, é o fato de que o grande número de situações e exemplos trabalhados pelo grupo proporcionou uma redução dos sintomas em um grande número de situações. Desta forma, destacou-se que a escolha das técnicas, influenciou uma intervenção visando o desenvolvimento de competências com o objetivo de generalização, para que os participantes pudessem aplicar os conhecimentos obtidos nos encontros terapêuticos em experiências de sua vida. Apesar da inserção de um grande número de situações, em que os indivíduos pudessem desenvolver suas habilidades, destacam-se tantas outras que não foram trabalhadas através da exposição, considerando as limitações de espaço, tempo e número de sessões. Além disso, muitas vezes as demandas de ansiedade social são provenientes das mais diversas situações sociais e de suas imprevisibilidades, torna-se inviável trabalhar cada situação específica, cabendo aos planos terapêuticos intervir em grupos de situações e possibilitar a desenvolvimento de habilidades que possam ser generalizadas para outros contextos.

Esta pesquisa apresentou 4 desistências, as quais comprometeram a dinâmica do grupo. Nesse caso, deve-se ter cuidado ao explanar sobre os resultados estatísticos, visto que os 9 integrantes que deram início ao grupo também não comportariam um número propício para ser analisado estatisticamente. Portanto, a pesquisa é uma descrição dos grupos e dos resultados dos participantes que finalizaram o tratamento. No entanto essa perda se mostrou significativa nos resultados finais, por diminuir a amostra. O protocolo utilizado nessa pesquisa/intervenção propõe número de sessões, tempo de duração e meios de tratamento (exposição, relaxamente, reestruturação e desenvolvimento de habilidades sociais). Foi possível perceber que os pontos de trabalho são bem completos e indispensáveis a quaisquer intervenções clínica com pacientes ansiosos sociais, seja individual ou 
em grupo. Abordar aspectos cognitivos e comportamentais, no que diz respeito às relações sociais e sintomas fisiológicos da ansiedade, é o mais indicado e avaliado positivamente pelos diversos estudos clínicos que a literatura traz. Porém, foi observado que muitos pacientes carecem de acompanhamento individual pós-grupo, o que pode ser um indicativo de tempo inadequado para nosso grupo amostral, comorbidades, ou ainda falta de adaptação das técnicas para uma intervenção de alcance mais profundo ou mudanças mais consistentes.

Portanto, entendemos que em nosso contexto de trabalho, o protocolo de Heimberg deve ser alterado em relação a número de sessões, e a equipe terapêutica deve utilizar esse tempo a mais para reforçar e consolidar as mudanças adquiridas inicialmente, uma vez que foram percebidas melhoras dos sintomas e alterações das crenças cognitivas negativas. Além disso, devido ao propósito de desenvolver um protocolo de tratamento, os próximos grupos terão como objeto ampliar a amostra do estudo e possivelmente demonstrar a significância estatística do modelo empregado. Tal processo traz avanços para o tratamento em grupo destinados aos pacientes ansiosos sociais, podendo futuramente servir de apoio para a melhoria da qualidade de vida destes pacientes. Neste contexto, será ampliado o número de grupos desenvolvidos anualmente, a fim de minimizar algumas limitações do estudo, tais como: a amostra reduzida, que inviabiliza uma análise estatística mais apurada, e dificuldades em comparar o protocolo com outros grupos desenvolvidos.

\section{Considerações finais}

A aprendizagem é um processo do conhecimento e de trocas constantes e ensinamentos que acompanham os indivíduos durante toda a sua vida. Desta forma, o presente projeto mostrou a importância da intervenção em grupo para pessoas com dificuldades de interação social e poderá servir de base para a elaboração de outros grupos terapêuticos, visando à maximização dos resultados e minimização dos sintomas que são experimentados com bastante sofrimento por aqueles que apresentam o TAS. Os pontos positivos evidenciados nos resultados, aliados à mudança de pensamento, as técnicas e atividades desenvolvidas, proporcionarão aos indivíduos dos novos grupos a oportunidade de serem assistidos com mais qualidade, otimizando o serviço oferecido bem como os resultados alcançados.

Devido às alterações apresentadas para o Transtorno de Ansiedade Social, no novo Manual Diagnóstico e Estatístico dos Transtornos Mentais (50 Edição), faz-se necessário uma adaptação das atividades 
desenvolvidas no grupo, principalmente para diminuir a ansiedade e o medo de uma avaliação negativa, bem como o comportamento de esquiva, um dos principais métodos de evitação deste transtorno, apontados com ênfase no DSM-5 (Associação Americana de Psiquiatria, 2014).

Os próximos grupos servirão para aumentar o número da amostra e diminuir os efeitos negativos das possíveis desistências. Para melhor avaliar as técnicas de intervenções mais eficazes e relevantes, serão realizados dois grupos com focos diferenciados, um com intervenções mais comportamentais e outro mais cognitivo, buscando assim comparar qual aspecto deve ser mais explorado clinicamente em um grupo de TCC para ansiosos sociais.

Torna-se necessário ampliar e aprofundar nos aspectos qualitativos das entrevistas e observações, visando reforçar aspectos relevantes que sirvam de subsídio para o desenvolvimento de futuras medidas psicométricas, e acrescentar uma avaliação de habilidades sociais, uma vez que é um constructo muito explorado no tratamento desse transtorno.

A TCC tem, cada vez mais, se mostrado eficaz para os transtornos de ansiedade (Manfro et al, 2008), e a TCCG tem se destacado em relação à TCC individual em casos de ansiedade social (D'el Rey \& Pacini, 2006). O grupo, além de compartilhar as experiências vividas, permite uma exposição e avaliações que aceleram as desconstruções de crenças disfuncionais, facilitando o processo de reestruturação cognitiva, e funcionando como apoio, ao motivar as situações de enfrentamento (Heimberg, 2002). Por isso, estudos sobre esta forma de abordagem têm sido cada vez mais relevantes e necessários para o fortalecimento da clínica em psicologia e intervenções terapêuticas.

\section{Referências}

Associação Psiquiátrica Americana. (2002). Manual Diagnóstico e Estatístico de Transtornos Mentais: Texto revisado - TR (4a ed.). Porto Alegre: Artmed.

Associação Psiquiátrica Americana (2014). Manual Diagnóstico e Estatístico de Transtornos Mentais ( $5 a$ ed.). Porto Alegre: Artmed.

Beck, J.S. (1997). Terapia Cognitiva: teoria e prática. Porto Alegre, RS: Artmed.

Barlow, D. H. (1999). Manual Clínico dos Transtornos Psicológicos. 2ạ ed. Porto Alegre: Artmed.

Barlow, H.D. (2002). Anxiety and its disorders: The nature and treatment of anxiety and panic. (2nd ed.). New York: Guilford Press. 
Caballo, V. E. (2012). Manual de Avaliação e Treinamento das Habilidades Sociais. (M. L. Marinho, Trad.). São Paulo: Santos. (Obra original publicada em 2003).

Cunha, J. A. (2001). Manual da versão em português das Escalas Beck. São Paulo: Casa do Psicólogo.

D'el Rey, G. J. F., \& Pacini, C. A. (2006). Terapia cognitivocomportamental da fobia social: modelos e técnicas. Psicologia em Estudo, Maringá, 11(2), 269-275.

D'el Rey, G. J. F, Greenberg, P. N., Husni, M. A., \& Cejkinski, A. (2007). Terapia cognitivo-comportamental de grupo no tratamento da fobia social generalizada. Psicologia Argumento, 25(50), 305-311.

Furmark, T. (2002). Social phobia: overview of community surveys. Acta Psychiatrica Scandinavica, 105, 84-93.

Heimberg, R. G. (2002) Cognitive-behavioral therapy for social anxiety disorder: current status and future directions. Biological Psychiatry, 51 Suppl 1: S101-8.

Hofmann, S. G. (2004). Cognitive mediation of treatment change in social phobia. Journal of Consulting and Clinical Psychology, 72(3), 392-399.

Hope, D. A., Heimberg, R. G., \& Turk, C. L. (2012). Terapia cognitivocomportamental para ansiedade social: Guia do terapeuta (2a ed.). Porto Alegre: Artmed.

Hudson, J. L., \& Rapee, R. M. (2000). The origins of Social Phobia. Behavior Modification, 24, 102-129.

Ito, L. M., Roso, M. C., Tiwari, S., Kendall, P. C., \& Asbahr, F. R. (2008). Terapia cognitivo-comportamental da fobia social. Revista Brasileira de Psiquiatria, 30(Supl. 2), 96-101.

Kessler, R. C., Berglund, P., Demler, O., J in, R., Merikangas, K. R., \& Walters E. E. (2005). Lifetime Prevalence and Age-of-Onset Distributions of DSM-IV Disorders in the National Comorbidity Survey Replication. Archives of General Psychiatry, 62, 593602.

Kessler, R. C., Gruber, M., Hettema, J. M., Hwang, I., Sampson, N., \& Yonkers, K. A. (2008). Co-morbid major depression and generalized anxiety disorders in the National Comorbidity Survey follow-up. Psychological Medicine, 38(3), 365-374.

Knijnik, D. Z. (2008). Fobia Social Generalizada: um estudo comparativo de duas modalidades terapêuticas. Tese de Doutorado, UFRS, Porto Alegre, Brasil.

Knijnik, D. Z., Kruter, B., Cordioli, A. V., \& Kapczinski, F. (2005). Tratamento Farmacológico na fobia social: diretrizes e algoritmo. Porto Alegre: Artmed.

Liebowitz, M. R., Gorman, J. M., Fyer, A. J., \& Klein, D. F. (1985). Social Phobia: Review of a Neglected Anxiety Disorder. Arch Gen Psychiatry, 42(7), 729-736. 
Manfro, G. G., Heldt, E., Cordioli, A. V., \& Otto, M. W. (2008) Terapia cognitivo-comportamental no transtorno de pânico. Revista Brasileira de Psiquiatria, 30(supl.2), S81-87.

Masia-Warner, C., Storch, E. A., Pincus, D. B., Klein, R. G., Heimberg, R. G., \& Liebowitz, M. R. (2003). The Liebowitz Social Anxiety Scale for Children and Adolescents: An Initial Psychometric Investigation. Journal of the American Academy of Child \& Adolescent Psychiatry, 42(9), 1076-1084.

Nardi, A. E. (2000). Transtorno de ansiedade social: fobia social - a timidez patológica. Rio de Janeiro: Medsi.

Neufeld, C. B., Moreira, C. A. M., \& Xavier, G. S. (2012). Terapia Cognitivo-Comportamental em Grupos de Emagrecimento: 0 Relato de Uma Experiência. Psico, 43(1), 93-100.

Nutt, C. \& Ballenger, J. (2010). Transtornos de Ansiedade: Transtorno de pânico e Transtorno de Ansiedade Social. Rio de Janeiro: Ed MedLine.

Pereira, S. M., \& Lourenço, L. M. (2012). Estudo bibliométrico do transtorno de ansiedade social em universitários. Arquivos Brasileiros de Psicologia, 64(1), 47-62.

Picon, P. (2006). Desenvolvimento da versão em Português do Social Phobia and Anxiety Inventory (SPAI) e estudos de fidedignidade e validade em amostras populacionais clínicas e brasileiras. Tese de Doutorado, UFRG, Porto Alegre, Brasil.

Picon, P., \& Knijinik, D. Z. (2008). Fobia Social. In Knapp, P. (Org.), Terapia Cognitivo-Comportamental na Prática Psiquiátrica. Porto Alegre: Artmed.

Pureza, J. R., Rusch, S. G. S., Wagner, M., \& Oliveira, M. S. (2012). Treinamento de Habilidades Sociais em Universitários: uma proposta de intervenção. Revista Brasileira de Terapias Cognitivas, 8(1), 2-9.

Ruscio, A. M., Brown. T. A., Chiu. W. T., Sareen. J., Stein. M. B., \& Kessler. R. C. (2008). Social fears and social phobia in the USA: results from the National Comorbidity Survey Replication. Psychological Medicine, 38(1), 15-28.

Santos, L. F. (2012). Estudo da validade e fidedignidade da Escala de Ansiedade Social de Liebowitz - versão auto-aplicada. Dissertação de Mestrado, Universidade de São Paulo, Ribeirão Preto, SP, Brasil.

Wang, P. S, Lane, M., Olfson, M., Pincus, H. A., Wells, K. B., \& Kessler, R. C. (2005). Twelve-month use of mental health services in the United States: results from the National Comorbidity Survey Replication. Archives of General Psychiatry, 62, 629-640.

Wright, J. H., Basco, M. R., \& Thase, M. E. (2008). Aprendendo a terapia cognitivo-comportamental: Um guia ilustrado. (M. G. 
Armando, Trad.). Porto Alegre: Artmed. (Obra original
publicada em 2006).

\section{Endereço para correspondência}

Carolina Pereira Dittz

Universidade Federal de Juiz de Fora, Juiz de Fora

Núcleo de Estudos em Violência e Ansiedade Social - NEVAS

Rua Santos Dumont, 214, Granbery, CEP 36010-386, Juiz de Fora - MG, Brasil

Endereço eletrônico: carolina.dittz@gmail.com

Francesca Stephan

Universidade Federal de Juiz de Fora

Núcleo de Estudos em Violência e Ansiedade Social - NEVAS

Rua Santos Dumont, 214, Granbery, CEP 36010-386, Juiz de Fora - MG, Brasil

Endereço eletrônico: francesca_st@yahoo.com.br

Daniel Alexandre Gouvêa Gömes

Universidade Federal de Juiz de Fora

Núcleo de Estudos em Violência e Ansiedade Social - NEVAS

Rua Santos Dumont, 214, Granbery, CEP 36010-386, Juiz de Fora - MG, Brasil

Endereço eletrônico: gouvea.dag@gmail.com

\section{Auxiliatrice Caneschi Badaró}

Universidade Federal de J uiz de Fora

Núcleo de Estudos em Violência e Ansiedade Social - NEVAS

Rua Santos Dumont, 214, Granbery, CEP 36010-386, Juiz de Fora - MG, Brasil

Endereço eletrônico: auxiliatrice@hotmail.com

\section{Lelio Moura Lourenço}

Universidade Federal de Juiz de Fora

Núcleo de Estudos em Violência e Ansiedade Social - NEVAS

Rua Santos Dumont, 214, Granbery, CEP 36010-386, Juiz de Fora - MG, Brasil

Endereço eletrônico: leliomlourenco@gmail.com

Recebido em: 16/02/2015

Reformulado em: 02/09/2015

Aceito para publicação em: 03/09/2015

\section{Notas}

* Mestre em Psicologia pela Universidade Federal de Juiz de Fora.

** Doutoranda em Psicologia pela Universidade Federal de Juiz de Fora.

*** Doutorando em Psicologia pela Universidade Federal de Juiz de Fora.

**** Professor Adjunto do Departamento de Psicologia da Universidade Federal de J uiz de Fora. 